\title{
Novel Global Exponential Stability Criterion for Recurrent Neural Networks with Time-Varying Delay
}

\author{
Wenguang Luo,, ${ }^{1,2}$ Xiuling Wang, ${ }^{1}$ Yonghua Liu, ${ }^{1}$ and Hongli Lan ${ }^{3}$ \\ ${ }^{1}$ School of Electrical and Information Engineering, Guangxi University of Science and Technology, Liuzhou 545006, China \\ ${ }^{2}$ Guangxi Key Laboratory of Automobile Components and Vehicle Technology, Guangxi University of Science and Technology, \\ Liuzhou 545006, China \\ ${ }^{3}$ School of Computer Engineering, Guangxi University of Science and Technology, Liuzhou 545006, China
}

Correspondence should be addressed to Wenguang Luo; wenguang.luo@163.com

Received 15 October 2012; Accepted 3 January 2013

Academic Editor: Massimo Furi

Copyright (C) 2013 Wenguang Luo et al. This is an open access article distributed under the Creative Commons Attribution License, which permits unrestricted use, distribution, and reproduction in any medium, provided the original work is properly cited.

The problem of global exponential stability for recurrent neural networks with time-varying delay is investigated. By dividing the time delay interval $[0, \tau(t)]$ into $K+1$ dynamical subintervals, a new Lyapunov-Krasovskii functional is introduced; then, a novel linear-matrix-inequality (LMI-) based delay-dependent exponential stability criterion is derived, which is less conservative than some previous literatures (Zhang et al., 2005; He et al., 2006; and Wu et al., 2008). An illustrate example is finally provided to show the effectiveness and the advantage of the proposed result.

\section{Introduction}

In the past decades, recurrent neural networks (RNNs) have been extensively investigated because of their applications, such as combinatorial optimization $[1,2]$, associative memories [3-5], signal processing [6], image processing [7], pattern recognition $[8,9]$, and so forth. Some of these applications often require that equilibrium points of the designed networks be stable. Meanwhile, in the hard implementation of RNNs, time delay commonly occurrs due to the finite switching speed of amplifiers or finite speed of signal processing, and its existence is always an origin of oscillation, divergence, and instability in neural networks. Therefore, the stability of RNNs with time delay has received much attention, and a large amount of results have been proposed to ensure the asymptotic or exponential stability of delayed neural networks [10-21].

So far, there is a main means handling the stability of delayed neural networks: free-weighting matrix approach
[22-26]. Recently, a novel method was proposed for Hopfield neural networks with constant delay in [27], which brings more free-weighting matrices by dividing equally the constant time delay interval $[0, \tau]$ into $m$ subintervals. Further more, by dividing the time delay interval $[0, \tau(t)]$ into $K+1$ dynamical subintervals, Zhang et al. [28] generalize this method to study global asymptotic stability of RNNs with time-varying delay. This method mainly utilizes the information in the time delay interval $[0, \tau(t)]$, which brings more freedom degrees and can reduce conservativeness.

Motivated by above-mentioned discussions, in this paper, we consider the global exponential stability of RNNs with time-varying delay. By dividing the time delay interval $[0, \tau(t)]$ into $K+1$ dynamical subintervals, we construct a new Lyapunov-Krasovskii functional (LKF) and derive a novel sufficient condition, which is presented in term of linear matrix inequality (LMI). The obtained stability result is less conservative than some existing results $[22,23,29]$. Finally, 
an illustrating example is given to verify the effectiveness and the advantage of the proposed result.

The rest of this paper is organized as follows. In Section 2, the problem of exponential stability analysis for RNNs with time-varying delay is formulated. Section 3 presents our main results. An illustrating example is provided in Section 4. The conclusion is stated in Section 5.

Throughout this paper, $C=\left[C_{i j}\right]_{n \times n}$ denotes an $n \times n$ real matrix. $C^{T},\|C\|, \lambda_{m}(C)$, and $\lambda_{M}(C)$ represent the transpose, the Euclidean norm, the minimum eigenvalue, and the maximum eigenvalue of matrix $C$, respectively. $C>0(C<$ 0 ) denotes that $C$ is a positive (negative) definite matrix. $I$ denotes an identify matrix with compatible dimensions, and * denotes the symmetric terms in a symmetric matrix.

\section{Problem Formulation}

Consider the following RNNs with time-varying delay:

$$
\begin{gathered}
\dot{z}(t)=-D z(t)+A f(z(t))+B f(z(t-\tau(t)))+J, \\
z(t)=\psi(t) \quad \forall t \in[-\tau, 0]
\end{gathered}
$$

where $z(\cdot)=\left[z_{1}(\cdot), z_{2}(\cdot), \ldots, z_{n}(\cdot)\right]^{T}$ is the state vector, $f(z(\cdot))=\left[f_{1}\left(z_{1}(\cdot)\right), f_{2}\left(z_{2}(\cdot)\right), \ldots, f_{n}\left(z_{n}(\cdot)\right)\right]^{T}$ denotes the neuron activation function, and $J$ is a bias value vector. $D=$ $\operatorname{diag}\left(d_{i}\right)$ is diagonal matrix with $d_{i}>0, i=1,2, \ldots, n$. $A$ and $B$ are connection weight matrix and the delay connection weight matrix, respectively. The initial condition $\psi(t)$ is a continuous and differentiable vector-valued function, where $t \in[-\tau, 0]$. The time delay $\tau(t)$ is a differentiable function that satisfies: $0 \leq \tau(t) \leq \tau, \dot{\tau}(t) \leq \mu$, where $\tau>0$ and $\mu \geq 0$.

To obtain the proposal result, we assume that each $f_{i}$ is bounded and satisfies

$$
l_{i}^{-} \leq \frac{f_{i}(u)-f_{i}(v)}{u-v} \leq l_{i}^{+}
$$

where $\forall u, v \in R, u \neq v . l_{i}^{-}$and $l_{i}^{+}$are some constants, $i=$ $1,2, \ldots, n$.

Let $g_{i}\left(z_{i}(t)\right)=f_{i}\left(z_{i}(t)\right)-l_{i}^{-} z_{i}(t), l_{i}=l_{i}^{+}-l_{i}^{-}$, system (1) is equivalent to the following form:

$$
\begin{aligned}
\dot{z}(t)= & -\left(D-A L_{0}\right) z(t)+A g(z(t))+B g(z(t-\tau(t))) \\
& +B L_{0} z(t-\tau(t))+J
\end{aligned}
$$

where $L_{0}=\operatorname{diag}\left(l_{1}^{-}, l_{2}^{-}, \ldots, l_{n}^{-}\right), L_{1}=\operatorname{diag}\left(l_{1}^{+}, l_{2}^{+}, \ldots, l_{n}^{+}\right), L=$ $\operatorname{diag}\left(l_{1}, l_{2}, \ldots, l_{n}\right)=L_{1}-L_{0}$.

Noting assumption (2), we have

$$
0 \leq \frac{g_{i}(u)-g_{i}(v)}{u-v} \leq l_{i}
$$

$\forall u, v \in R, u \neq v, l_{i}=l_{i}^{+}-l_{i}^{-}(i=1,2, \ldots, n)$.
Assuming system (3) has an equilibrium point $z^{*}=$ $\left(z_{1}^{*}, z_{2}^{*}, \ldots, z_{n}^{*}\right)^{T}$. Then, let $x=\left(x_{1}(t), x_{2}(t), \ldots, x_{n}(t)\right)^{T}$, and we define $x_{i}(t)=z_{i}(t)-z_{i}^{*}$, system (3) is transformed into the following form:

$$
\begin{aligned}
\dot{x}(t)= & -\left(D-A L_{0}\right) x(t)+A \bar{g}(x(t)) \\
& +B \bar{g}(x(t-\tau(t)))+B L_{0} x(t-\tau(t)),
\end{aligned}
$$

where $\bar{g}_{i}\left(x_{i}(t)\right)=g_{i}\left(x_{i}(t)+z_{i}^{*}\right)-g_{i}\left(z_{i}^{*}\right)$ with $\bar{g}_{i}(0)=$ $0, \bar{g}_{i}\left(x_{i}(t-\tau(t))\right)=g_{i}\left(x_{i}(t-\tau(t))+z_{i}^{*}\right)-g_{i}\left(z_{i}^{*}\right)$.

In the derivation of the main results, we need the following lemmas and definitions.

Definition 1 (global exponential stability). System (5) is said to be globally exponentially stable with convergence rate $k$, if there exist constants $k>0$ and $M \geq 1$, such that

$$
\|x(t)\| \leq M \phi e^{-k t}, \quad \forall t \geq 0
$$

where $\phi=\sup _{-\tau \leq \theta \leq 0}\|x(\theta)\|$.

Lemma 2. Let $x(t) \in R^{n}$ be a vector-valued function with the first-order continuous-derivative entries. Then, the following integral inequality holds for matrix $X=X^{T}>0$ and any matrices $M_{1}, M_{2}$, and two scalar functions $h_{1}(t)$ and $h_{2}(t)$, where $h_{2}(t) \geq h_{1}(t) \geq 0$

$$
\begin{aligned}
& -\int_{t-h_{2}(t)}^{t-h_{1}(t)} \dot{x}^{T}(s) X \dot{x}(s) d s \\
& \quad \leq \zeta^{T}(t)\left[\begin{array}{cc}
M_{1}^{T}+M_{1} & -M_{1}^{T}+M_{2} \\
* & -M_{2}^{T}-M_{2}
\end{array}\right] \zeta(t) \\
& \quad+\left(h_{1}(t)-h_{2}(t)\right) \zeta^{T}(t) H^{T} R^{-1} H \zeta(t),
\end{aligned}
$$

where $H=\left[M_{1}, M_{2}\right]_{T} \in R^{n \times 2 n}$ and $\zeta(t)=$ $\left[x^{T}\left(t-h_{1}(t)\right) x^{T}\left(t-h_{2}(t)\right)\right]^{T}$.

Proof. This proof can be completed in a manner similar to [30].

Lemma 3 (see [31]). For any two vectors $a, b \in R^{n}$, any matrix $A$, any positive definite symmetric matrix $B$ with the same dimensions, and any two positive constants $m, n$, the following inequality holds:

$$
-m a^{T} B a+2 n a^{T} A b \leq n^{2} b^{T} A^{T}(m B)^{-1} A b .
$$

\section{Main Results}

In this section, we will consider the delay interval $[0, \tau(t)]$, which is divided into $K+1$ dynamical subintervals, namely, $\left[0, \rho_{1} \tau(t)\right], \ldots,\left[\rho_{K} \tau(t), \tau(t)\right]$, where $\rho_{1}<\cdots<\rho_{K}$. This is to 
say, there is a parameter sequence $\left(\rho_{1}, \ldots, \rho_{K}\right)$, which satisfies the following conditions:

$$
\begin{gathered}
0<\rho_{1} \tau(t)<\rho_{2} \tau(t)<\cdots<\rho_{K} \tau(t)<\tau(t), \\
0 \leq \rho_{i} \dot{\tau}(t) \leq \rho_{i} \mu,
\end{gathered}
$$

where $\rho_{i} \in(0,1), i=1,2, \ldots, K, K$ is positive integer.

Utilizing the useful information of $K+1$ dynamical subintervals, a novel LKF is constructed, and then a newly LMIbased delay-dependent sufficient condition can be proposed to guarantee the global exponential stability of RNNs with time-varying delay.

Theorem 4. The equilibrium point of system (5) with $\mu<1$ is globally exponentially stable with convergence rate $k>0$, if there exists parameter $\rho_{i}$ satisfying $0<\rho_{1}<\cdots<\rho_{K}<$ 1 , some positive definite symmetric matrices $P, R_{1}, R_{2}, R_{3}$,
$Q_{i}, Z$, some positive definite diagonal matrices $\Lambda, X_{1}, X_{2}, Y_{1}$ and $Y_{2}$, and any matrices $M_{j}$, where $i=1,2, \ldots, K, j=$ $1,2, \ldots, 2 K+4$, and $K$ is a positive integer, such that the following LMI has feasible solution:

$$
\left[\begin{array}{ccccc}
\Sigma & W_{0} & 0 & 0 & 0 \\
* & \frac{-Z}{\rho_{1}} & W_{1} & 0 & 0 \\
* & * & \frac{-Z}{\rho_{2}-\rho_{1}} & \cdots & 0 \\
* & * & * & \cdots & W_{K} \\
* & * & * & * & \frac{-Z}{1-\rho_{K}}
\end{array}\right]<0,
$$

where $W_{i}=\tau e^{-k \tau} H_{i}^{T}, H_{i}=\left[\begin{array}{ll}M_{2 i+1} & M_{2 i+2}\end{array}\right] \in R^{n \times 2 n}, i=$ $0,1,2, \ldots, K$.

$$
\left.\begin{array}{cccc}
\Sigma_{1, K+2} & 0 & \Sigma_{1, K+4} & \Sigma_{1, K+5} \\
0 & 0 & 0 & 0 \\
0 & 0 & 0 & 0 \\
0 & 0 & 0 & 0 \\
0 & 0 & 0 & 0 \\
\Sigma_{K+1, K+2} & 0 & 0 & 0 \\
\Sigma_{K+2, K+2} & \Sigma_{K+2, K+3} & \Sigma_{K+2, K+4} & \Sigma_{K+2, K+5} \\
* & \Sigma_{K+3, K+3} & 0 & 0 \\
* & * & \Sigma_{K+4, K+4} & \Sigma_{K+4, K+5} \\
* & * & * & \Sigma_{K+5, K+5}
\end{array}\right],
$$

$$
\begin{aligned}
\Sigma_{1,1}= & 2 k P-P\left(D-A L_{0}\right)-\left(D-A L_{0}\right)^{T} P \\
& +R_{1}+R_{3}+\tau^{2}\left(D-A L_{0}\right)^{T} Z\left(D-A L_{0}\right) \\
& +\sum_{i=1}^{K} Q_{i}+L X_{1} L+\tau e^{-2 k \tau}\left(M_{1}^{T}+M_{1}\right), \\
\Sigma_{1,2}= & \tau e^{-2 k \tau}\left(-M_{1}^{T}+M_{2}\right), \\
\Sigma_{1, K+2}= & P B L_{0}-\tau^{2}\left(D-A L_{0}\right) Z B L_{0}, \\
\Sigma_{1, K+4}= & P A+2 k \Lambda-\left(D-A L_{0}\right)^{T} \Lambda \\
& -\tau^{2}\left(D-A L_{0}\right)^{T} Z A+L X_{2}, \\
\Sigma_{1, K+5}= & P B-\tau^{2}\left(D-A L_{0}\right)^{T} Z B, \\
\Sigma_{2,2}= & -e^{-2 k \rho_{1} \tau} Q_{1}\left(1-\rho_{1} \mu\right)+\tau e^{-2 k \tau}\left(-M_{2}^{T}-M_{2}\right) \\
+ & \tau e^{-2 k \tau}\left(M_{3}^{T}+M_{3}\right), \\
\Sigma_{2,3}= & \tau e^{-2 k \tau}\left(-M_{3}^{T}+M_{4}\right), \\
\Sigma_{3,3}= & -e^{-2 k \rho_{2} \tau} Q_{2}\left(1-\rho_{2} \mu\right)+\tau e^{-2 k \tau}\left(-M_{4}^{T}-M_{4}\right) \\
+ & \tau e^{-2 k \tau}\left(M_{5}^{T}+M_{5}\right),
\end{aligned}
$$

$$
\begin{aligned}
\Sigma_{3,4}=\tau e^{-2 k \tau}\left(-M_{5}^{T}+M_{6}\right), \ldots, & \\
\Sigma_{K+1, K+1}= & -e^{-2 k \rho_{K} \tau} Q_{K}\left(1-\rho_{K} \mu\right) \\
& +\tau e^{-2 k \tau}\left(-M_{2 K}^{T}-M_{2 K}\right) \\
& +\tau e^{-2 k \tau}\left(M_{2 K+1}^{T}+M_{2 K+1}\right), \\
\Sigma_{K+1, K+2}= & \tau e^{-2 k \tau}\left(-M_{2 K+1}^{T}+M_{2 K+2}\right), \\
\Sigma_{K+2, K+2}= & -e^{-2 k \tau} R_{1}(1-\mu) \\
& +\tau^{2} L_{0} B^{T} Z B L_{0}+L Y_{1} L \\
& +\tau e^{-2 k \tau}\left(-M_{2 K+2}^{T}-M_{2 K+2}\right) \\
& +\tau e^{-2 k \tau}\left(M_{2 K+3}^{T}+M_{2 K+3}\right) \\
\Sigma_{K+2, K+3} & \tau e^{-2 k \tau}\left(-M_{2 K+3}^{T}+M_{2 K+4}\right), \\
\Sigma_{K+2, K+4}= & L_{0} B^{T} \Lambda+\tau^{2} L_{0} B^{T} Z A, \\
\Sigma_{K+2, K+5}= & \tau^{2} L_{0} B^{T} Z B+L Y_{2}, \\
\Sigma_{1} &
\end{aligned}
$$




$$
\begin{aligned}
& \Sigma_{K+3, K+3}= e^{-2 k \tau} R_{3}+\tau e^{-2 k \tau}\left(-M_{2 K+4}^{T}-M_{2 K+4}\right), \\
& \Sigma_{K+4, K+4}= \Lambda A+A^{T} \Lambda+R_{2} \\
&+\tau^{2} A^{T} Z A-X_{1}-2 X_{2}, \\
& \Sigma_{K+4, K+5}= \Lambda B+\tau^{2} A^{T} Z B \\
& \Sigma_{K+2, K+5}=-e^{-2 k \tau} R_{2}(1-\mu) \\
&+\tau^{2} B^{T} Z B-Y_{1}-2 Y_{2}, \\
& L_{0}=\operatorname{diag}\left(l_{i}^{-}\right), \\
& L_{1}=\operatorname{diag}\left(l_{i}^{+}\right), \\
& L_{0}=\operatorname{diag}\left(l_{i}\right), \\
& D=\operatorname{diag}\left(d_{i}\right), \\
& A=\left[a_{i j}\right]_{n \times n} \\
& B=\left[b_{i j}\right]_{n \times n}
\end{aligned}
$$

Proof. Construct the following Lyapunov-Krasovskii functional candidate:

$$
\begin{aligned}
V(x(t))= & V_{1}(x(t))+V_{2}(x(t))+V_{3}(x(t)) \\
& +V_{4}(x(t))+V_{5}(x(t)),
\end{aligned}
$$

where

$$
\begin{aligned}
V_{1}(x(t))= & e^{2 k t} x^{T}(t) P x(t), \\
V_{2}(x(t))= & 2 e^{2 k t} \sum_{i=1}^{n} \lambda_{i} \int_{0}^{x_{i}(t)} \bar{g}_{i}(s) d s, \\
V_{3}(x(t))= & \int_{t-\tau(t)}^{t} e^{2 k s} x^{T}(s) R_{1} x(s) d s \\
& +\int_{t-\tau(t)}^{t} e^{2 k s} \bar{g}^{T}(s) R_{2} \bar{g}(s) d s \\
& +\int_{t-\tau}^{t} e^{2 k s} x^{T}(s) R_{3} x(s) d s, \\
V_{4}(x(t))= & \sum_{i=1}^{K} \int_{t-\rho_{i} \tau(t)}^{t} e^{2 k s} x^{T}(s) Q_{i} x(s) d s, \\
V_{5}(x(t))= & \tau \int_{-\tau}^{0} \int_{t+\theta}^{t} e^{2 k s} \dot{x}^{T}(s) Z \dot{x}(s) d s,
\end{aligned}
$$

where $P=P^{T}>0, \Lambda=\operatorname{diag}\left(\lambda_{i}\right)>0, R_{1}=R_{1}^{T}>0, R_{2}=$ $R_{2}^{T}>0, R_{3}=R_{3}^{T}>0, Q_{i}=Q_{i}^{T}>0, Z=Z^{T}>0$, and $i=0,1,2, \ldots, K$.
Let the parameters $\rho_{0}=0, \rho_{K+1}=1$; calculating the time derivatives $V_{i}(i=1,2,3,4,5)$ along the trajectories of system (5) yields

$$
\begin{aligned}
& \dot{V}_{1}(x(t))=2 k e^{2 k t} x^{T}(t) P x(t)+2 e^{2 k t} x^{T}(t) P \dot{x}(t), \\
& \dot{V}_{2}(x(t))=4 k e^{2 k t} \sum_{i=1}^{n} \lambda_{i} \int_{0}^{x_{i}(t)} \bar{g}_{i}(s) d s \\
& +2 e^{2 k t} \bar{g}^{T}(x(t)) \Lambda \dot{x}(t), \\
& \dot{V}_{3}(x(t))=e^{2 k t}\left[x^{T}(t) R_{1} x(t)\right. \\
& -e^{-2 k \tau(t)} x^{T}(t-\tau(t)) R_{1} x(t-\tau(t)) \\
& \times(1-\dot{\tau}(t)) \\
& +\bar{g}^{T}(x(t)) R_{2} \bar{g}(x(t)) \\
& -e^{-2 k \tau(t)} \bar{g}^{T}(x(t-\tau(t))) R_{2} \bar{g}(x(t-\tau(t))) \\
& \times(1-\dot{\tau}(t))+x^{T}(t) R_{3} x(t) \\
& \left.-e^{-2 k \tau} \bar{g}^{T}(x(t-\tau)) R_{3} \bar{g}(x(t-\tau))\right],
\end{aligned}
$$

$\dot{V}_{4}(x(t))=\sum_{i=1}^{K} e^{2 k t}\left[x^{T}(t) Q_{i} x(t)-e^{-2 k \rho_{i} \tau(t)} x^{T}\left(t-\rho_{i} \tau(t)\right)\right.$

$$
\left.\times Q_{i} x\left(t-\rho_{i} \tau(t)\right)\left(1-\rho_{i} \dot{\tau}(t)\right)\right],
$$

$\dot{V}_{5}(x(t))=\tau^{2} e^{2 k t} \dot{x}^{T}(t) Z \dot{x}(t)-\tau \int_{t-\tau}^{t} e^{2 k s} \dot{x}^{T}(s) Z \dot{x}(s) d s$.

It is clear that the following inequality is true:

$$
\begin{aligned}
\dot{x}^{T}(t) Z \dot{x}(t) \leq & {\left[-\left(D-A L_{0}\right) x(t)+A \bar{g}(x(t))\right.} \\
+ & B \bar{g}(x(t-\tau(t)))+B x(t-\tau(t))]^{T} \\
\times & Z\left[-\left(D-A L_{0}\right) x(t)+A \bar{g}(x(t))\right. \\
& +B \bar{g}(x(t-\tau(t)))+B x(t-\tau(t))],
\end{aligned}
$$

According to (4), for some diagonal matrices $X_{1}>0, X_{2}>$ $0, Y_{1}>0, Y_{2}>0$, we have

$$
\begin{gathered}
\bar{g}^{T}(x(t)) X_{1} \bar{g}(x(t)) \leq x^{T}(t) L X_{1} L x(t), \\
\bar{g}^{T}(x(t)) X_{2} \bar{g}(x(t)) \leq x^{T}(t) L X_{1} \bar{g}(x(t)), \\
\bar{g}^{T}(x(t-\tau(t))) Y_{1} \bar{g}(x(t-\tau(t))) \\
\leq x^{T}(t-\tau(t)) L Y_{1} L x(t-\tau(t)), \\
\bar{g}^{T}(x(t-\tau(t))) Y_{2} \bar{g}(x(t-\tau(t))) \\
\leq x^{T}(t-\tau(t)) L Y_{2} \bar{g}(x(t-\tau(t))) .
\end{gathered}
$$


Using Lemma 2, we have

$$
\begin{aligned}
& -\int_{t-\rho_{i+1} \tau(t)}^{t-\rho_{i} \tau(t)} \dot{x}^{T}(s) Z \dot{x}(s) d s \\
& \quad \leq \zeta_{i}^{T}(t)\left[\begin{array}{cc}
M_{2 i+1}^{T}+M_{2 i+1} & -M_{2 i+1}^{T}+M_{2 i+2} \\
* & -M_{2 i+2}^{T}-M_{2 i+2}
\end{array}\right] \zeta_{i}(t) \\
& \quad+\left(\rho_{i+1}-\rho_{i}\right) \tau(t) \zeta_{i}^{T}(t) H_{i}^{T} Z^{-1} H_{i} \zeta_{i}(t) \\
& \quad \int_{t-\tau}^{t-\tau(t)} \dot{x}^{T}(s) Z \dot{x}(s) d s \\
& \quad \leq \zeta_{K+1}^{T}(t)\left[\begin{array}{cc}
M_{2 K+3}^{T}+M_{2 K+3} & -M_{2 K+3}^{T}+M_{2 K+4} \\
* & -M_{2 K+4}^{T}-M_{2 K+4}
\end{array}\right] \zeta_{K+1}(t),
\end{aligned}
$$

where $H_{i}=\left[\begin{array}{ll}M_{2 i+1} & M_{2 i+2}\end{array}\right] \in R^{n \times 2 n}, \zeta_{i}(t)=$ $\left[\begin{array}{lll}x^{T}\left(t-\rho_{i} \tau(t)\right) & x^{T}\left(t-\rho_{i+1} \tau(t)\right)\end{array}\right]^{T}, \quad \zeta_{K+1}(t)=$ $\left[x^{T}(t-\tau(t)) x^{T}(t-\tau)\right]^{T}, i=0,1, \ldots, K$.

From (15)-(17), and using (18), we finally get

$$
\dot{V}(x(t)) \leq e^{2 k t} \Omega,
$$

where $\Omega=\zeta^{T}(t) \Sigma \zeta(t)+\Omega_{0}+\Omega_{1}+\cdots+\Omega_{K}, \Sigma$ is defined in (11).

$$
\begin{gathered}
\Omega_{i}=\left(\rho_{i+1}-\rho_{i}\right) \tau^{2} e^{-2 k \tau} \zeta_{i}^{T}(t) H_{i}^{T} Z^{-1} H_{i} \zeta_{i}(t), \\
i=0,1, \ldots, K, \\
\zeta_{j}(t)=\left[x^{T}\left(t-\rho_{j} \tau(t)\right) x^{T}\left(t-\rho_{j+1} \tau(t)\right)\right]^{T}, \\
j=0,1, \ldots, K+1 . \\
\zeta(t)=\left[x^{T}(t), x^{T}\left(t-\rho_{1} \tau(t)\right), \ldots, x^{T}\left(t-\rho_{K} \tau(t)\right),\right. \\
x^{T}(t-\tau(t)), x^{T}(t-\tau), \\
\left.\bar{g}^{T}(x(t)), \bar{g}^{T}(x(t-\tau(t)))\right]^{T} .
\end{gathered}
$$

Obviously, if $\Omega<0$, it implies $\dot{V}(x(t))<0$ for any $\zeta(t) \neq 0$.

And

$$
\begin{aligned}
V(x(0))= & x^{T}(0) P x(0)+2 \sum_{i=1}^{n} \lambda_{i} \int_{0}^{x_{i}(0)} \bar{g}_{i}(s) d s \\
& +\int_{-\tau(0)}^{0} e^{2 k s} x^{T}(s) R_{1} x(s) d s \\
& +\int_{-\tau(0)}^{0} e^{2 k s} \bar{g}^{T}(x(s)) R_{2} \bar{g}(x(s)) d s \\
& +\int_{-\tau}^{0} e^{2 k s} x^{T}(s) R_{3} x(s) d s \\
& +\sum_{i=1}^{K} \int_{-\rho_{i} \tau(0)}^{0} e^{2 k s} x^{T}(s) Q_{i} x(s) d s \\
& +\tau \int_{-\tau}^{0} \int_{\theta}^{0} e^{2 k s} \dot{x}^{T}(s) Z \dot{x}(s) d s .
\end{aligned}
$$

On the other hand, the following inequality holds:

$$
\begin{aligned}
\dot{x}^{T}(s) \dot{x}(s) \leq & {\left[-\left(D-A L_{0}\right) x(s)+A \bar{g}(x(s))\right.} \\
& +B \bar{g}(x(t-\tau(s)))+B x(t-\tau(s))]^{T} \\
\times & {\left[-\left(D-A L_{0}\right) x(s)+A \bar{g}(x(s))\right.} \\
& +B \bar{g}(x(t-\tau(s)))+B x(t-\tau(s))] .
\end{aligned}
$$

Combining Lemma 3, we have

$$
\begin{aligned}
\dot{x}^{T}(s) \dot{x}(s) \leq 4[ & \lambda_{M}\left(\left(D-A L_{0}\right)^{T}\left(D-A L_{0}\right)\right) \\
& +\lambda_{M}\left(L^{2}\right) \lambda_{M}\left(A^{T} A\right) \\
& \left.+\lambda_{M}\left(L^{2}\right) \lambda_{M}\left(B^{T} B\right)+\lambda_{M}\left(L_{0} B^{T} B L_{0}\right)\right]\|\phi\| .
\end{aligned}
$$

Thus,

$$
\begin{aligned}
V(x(0))= & \lambda_{M}(P)\|\phi\|^{2}+\left(\lambda_{M}\left(L^{2}\right)+\lambda_{M}\left(\Lambda^{T} \Lambda\right)\right)\|\phi\|^{2} \\
& +\lambda_{M}\left(R_{1}\right) \tau\|\phi\|^{2}+\lambda_{M}\left(R_{2}\right) \lambda_{M}\left(L^{2}\right) \tau\|\phi\|^{2} \\
& +\lambda_{M}\left(R_{3}\right) \tau\|\phi\|^{2}+\sum_{i=1}^{K} \lambda_{M}\left(Q_{i}\right) \rho_{i} \tau\|\phi\|^{2} \\
& +2 \tau^{3} \lambda_{M}(Z)\left[\lambda_{M}\left(\left(D-A L_{0}\right)^{T}\left(D-A L_{0}\right)\right)\right. \\
& +\lambda_{M}\left(L^{2}\right) \lambda_{M}\left(A^{T} A\right) \\
& +\lambda_{M}\left(L^{2}\right) \lambda_{M}\left(B^{T} B\right) \\
& \left.+\lambda_{M}\left(L_{0} B^{T} B L_{0}\right)\right]\|\phi\|
\end{aligned}
$$

where

$$
\begin{aligned}
\Delta= & \lambda_{M}(P)+\left(\lambda_{M}\left(L^{2}\right)+\lambda_{M}\left(\Lambda^{T} \Lambda\right)\right)+\lambda_{M}\left(R_{1}\right) \tau \\
& +\lambda_{M}\left(R_{2}\right) \lambda_{M}\left(L^{2}\right) \tau+\lambda_{M}\left(R_{3}\right) \tau \\
& +\sum_{i=1}^{K} \lambda_{M}\left(Q_{i}\right) \rho_{i} \tau\|\phi\|^{2} \\
& +2 \tau^{3} \lambda_{M}(Z)\left[\lambda_{M}\left(\left(D-A L_{0}\right)^{T}\left(D-A L_{0}\right)\right)\right. \\
& +\lambda_{M}\left(L^{2}\right) \lambda_{M}\left(A^{T} A\right) \\
& \left.+\lambda_{M}\left(L^{2}\right) \lambda_{M}\left(B^{T} B\right)+\lambda_{M}\left(L_{0} B^{T} B L_{0}\right)\right] .
\end{aligned}
$$

On the other hand, we have

$$
V(x(t)) \geq e^{2 k t} \lambda_{m}(P)\|\phi\| .
$$

Therefore,

$$
\|x(t)\| \leq e^{-k t} \sqrt{\frac{\Delta}{\lambda_{m}(P)}}\|\phi\| .
$$


Thus, according to Definition 1, we can conclude that the equilibrium point $x^{*}$ of system (5) is globally exponentially stable. This completes the proof.

Remark 5. Differential from the results in $[22,23,29]$, we divide the time delay interval $[0, \tau(t)]$ into $K+1$ dynamical subintervals, and a novel Lyapunov-Krasovskii functional is introduced. This brings more degrees of freedom to ensure the global exponential stability. Therefore, Theorem 4 is less conservative than some previous results.

Remark 6. In Theorem 4, by setting $R_{1}=R_{2}=Q_{i}=0(i=$ $1,2, \ldots, K)$, similar to the proof of Theorem 4 , we can derive a criterion to guarantee the global exponential stability of RNNs with time-varying delay when $\dot{\tau}(t)$ is unknown or $\tau(t)$ is not differentiable.

\section{Illustrating Example}

In this section, an illustrating example is given to verify the effectiveness and advantage of the criteria proposed in this paper.

Example 7. Consider system (5) with the following parameters:

$$
\begin{aligned}
D & =\left[\begin{array}{cc}
2 & 0 \\
0 & 3.5
\end{array}\right], \\
A & =\left[\begin{array}{cc}
-1 & 0.5 \\
0.5 & -1
\end{array}\right], \\
B & =\left[\begin{array}{cc}
-0.5 & 0.5 \\
0.5 & 0.5
\end{array}\right], \\
L & =\left[\begin{array}{ll}
1 & 0 \\
0 & 0
\end{array}\right] .
\end{aligned}
$$

At first, we suppose that the time delay interval $[0, \tau(t)]$ is divided into $2(K=1)$ subintervals, and $\rho=0.1$. While the upper bound $\tau=1$, the exponential convergence rates for various $\mu$ obtained from Theorem 4 and those in [22, 23, 29] are listed in Table 1. In addition, while the exponential convergence rate of $k=0.8$, the upper bounds of $\tau$ for various $\mu$ from Theorem 4 and those in $[22,23,29]$ are listed in Table 2.

Thus, from Tables 1 and 2, we can say that, the result in this paper is much effective and less conservative than those in $[22,23,29]$. Figure 1 shows the state response of Example 7 with constant delay $\tau=1$, when the initial value is $[0.8,-0.6]^{T}$.

\section{Conclusion}

In this paper, we consider the global exponential stability for RNNs with time-varying delay. By dividing the time delay interval $[0, \tau(t)]$ into $K+1$ dynamical subintervals, a novel Lyapunov-Krasovskii functional is introduced. A less conservative LMI-based delay-dependent stability criterion is derived based on Lyapunov stability theory. Furthermore, an

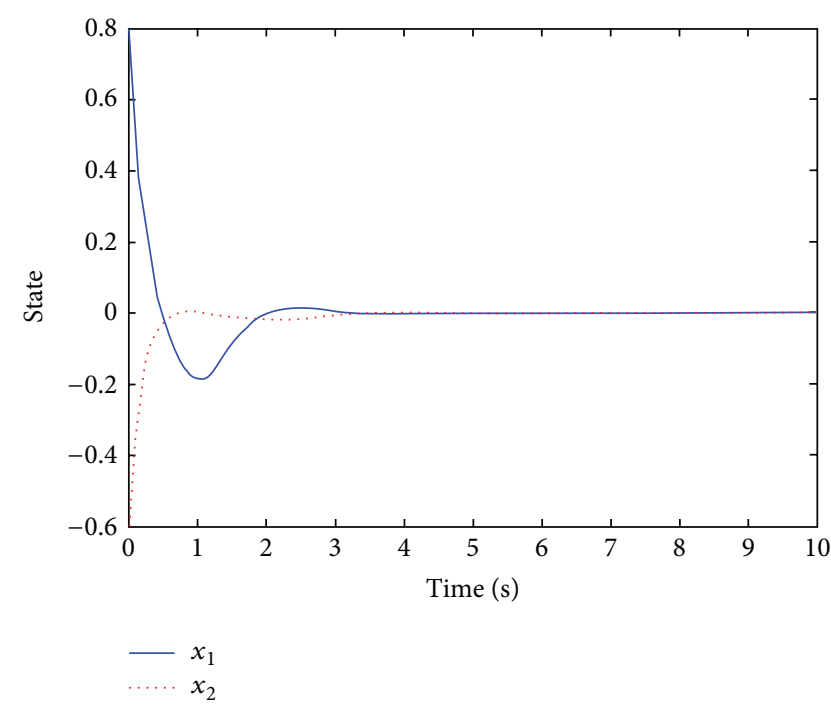

FIGURE 1: State response curves with $\tau=1$ and $\mu=0$ for Example 7 .

TABLE 1: Allowable exponential convergence rate $k$ for variable $\mu$ and $\tau=1$.

\begin{tabular}{lcccc}
\hline$\mu$ & 0 & 0.5 & 0.9 & Unsure \\
\hline$[22]$ & 0.25 & Fail & Fail & Fail \\
{$[23]$} & 1.15 & 0.7538 & 0.6106 & 0.3391 \\
{$[29]$} & 1.15 & 0.8643 & 0.8344 & 0.8169 \\
Theorem 4 $(\rho=1)$ & 1.15 & 0.9105 & 0.9105 & 0.8608 \\
\hline
\end{tabular}

TABLE 2: Allowable upper bound of $\tau$ for variable $\mu$ and $k=0.8$.

\begin{tabular}{lccc}
\hline$\mu$ & 0.5 & 0.8 & Unsure \\
\hline$[22]$ & Fail & Fail & Fail \\
{$[23]$} & 1.2606 & 0.9442 & 0.8310 \\
{$[29]$} & 1.2787 & 1.0819 & 1.0366 \\
Theorem 4 $(\rho=1)$ & 1.3022 & 1.1646 & 1.0817 \\
\hline
\end{tabular}

illustrating example is given to show the effectiveness of the proposed result.

\section{Acknowledgments}

This work is supported by Guangxi Science Foundation Grant (0832067), the Foundation Grant of Guangxi Key Laboratory of Automobile Components and Vehicle Technology (13-A03-01), and the Opening Project of Guangxi Key Laboratory of Automobile Components and Vehicle Technology (2012KFZD03).

\section{References}

[1] Y. H. Chen and S. C. Fang, "Solving convex programming problems with equality constraints by neural networks," Computers and Mathematics with Applications, vol. 36, no. 7, pp. 41-68, 1998. 
[2] Y. H. Chen and S. C. Fang, "Neurocomputing with time delay analysis for solving convex quadratic programming problems," IEEE Transactions on Neural Networks, vol. 11, no. 1, pp. 230240, 2000.

[3] J. A. Farrell and A. N. Michel, "A synthesis procedure for Hopfield's continuous-time associative memory," IEEE Transactions on Circuits and Systems, vol. 37, no. 7, pp. 877-884, 1990.

[4] A. N. Michel, J. A. Farrell, and H. F. Sun, "Analysis and synthesis techniques for Hopfield type synchronous discrete time neural networks with application to associative memory," IEEE Transactions on Circuits and Systems, vol. 37, no. 11, pp. 1356-1366, 1990.

[5] T. Nishikawa, Y. C. Lai, and F. C. Hoppensteadt, "Capacity of oscillatory associative-memory networks with error-free retrieval," Physical Review Letters, vol. 92, no. 10, Article ID 108101, 4 pages, 2004.

[6] L. O. Chua and L. Yang, "Cellular neural networks: applications," IEEE Transactions on Circuits and Systems, vol. 35, no. 10, pp. 1273-1290, 1988.

[7] R. Cancelliere, M. Gai, and A. Slavova, "Application of polynomial cellular neural networks in diagnosis of astrometric chromaticity," Applied Mathematical Modelling, vol. 34, no. 12, pp. 4243-4252, 2010.

[8] S. S. Young, P. D. Scott, and N. M. Nasrabadi, "Object recognition using multilayer Hopfield neural network," IEEE Transactions on Image Processing, vol. 6, no. 3, pp. 357-372, 1997.

[9] L. Chen, W. Xue, and N. Tokuda, "Classification of 2dimensional array patterns: assembling many small neural networks is better than using a large one," Neural Networks, vol. 23, no. 6, pp. 770-781, 2010.

[10] L. Olien and J. Bélair, "Bifurcations, stability, and monotonicity properties of a delayed neural network model," Physica D, vol. 102, no. 3-4, pp. 349-363, 1997.

[11] S. Arik, "An analysis of global asymptotic stability of delayed cellular neural networks," IEEE Transactions on Neural Networks, vol. 13, no. 5, pp. 1239-1242, 2002.

[12] J. Cao and D. Zhou, "Stability analysis of delayed cellular neural networks," Neural Networks, vol. 11, no. 9, pp. 1601-1605, 1998.

[13] J. Wei and S. Ruan, "Stability and bifurcation in a neural network model with two delays," Physica D, vol. 130, no. 3-4, pp. 255-272, 1999.

[14] X. Liao, G. Chen, and E. N. Sanchez, "Delay-dependent exponential stability analysis of delayed neural networks: an LMI approach," Neural Networks, vol. 15, no. 7, pp. 855-866, 2002.

[15] H. Huang, J. Cao, and J. Wang, "Global exponential stability and periodic solutions of recurrent neural networks with delays," Physics Letters A, vol. 298, no. 5-6, pp. 393-404, 2002.

[16] B. Chen and J. Wang, "Global exponential periodicity and global exponential stability of a class of recurrent neural networks with various activation functions and time-varying delays," Neural Networks, vol. 20, no. 10, pp. 1067-1080, 2007.

[17] H. Jiang and Z. Teng, "Global eponential stability of cellular neural networks with time-varying coefficients and delays," Neural Networks, vol. 17, no. 10, pp. 1415-1425, 2004.

[18] S. Senan and S. Arik, "New results for exponential stability of delayed cellular neural networks," IEEE Transactions on Circuits and Systems II, vol. 52, no. 3, pp. 154-158, 2005.

[19] Q. Zhang, X. Wei, and J. Xu, "An analysis on the global asymptotic stability for neural networks with variable delays," Physics Letters A, vol. 328, no. 2-3, pp. 163-169, 2004.
[20] H. Zhao and G. Wang, "Delay-independent exponential stability of recurrent neural networks," Physics Letters A, vol. 333, no. 5-6, pp. 399-407, 2004.

[21] T. L. Liao, J. J. Yan, C. J. Cheng, and C. C. Hwang, "Globally exponential stability condition of a class of neural networks with time-varying delays," Physics Letters A, vol. 339, no. 3-5, pp. 333342, 2005.

[22] Q. Zhang, X. Wei, and J. Xu, "Delay-dependent exponential stability of cellular neural networks with time-varying delays," Chaos, Solitons \& Fractals, vol. 23, no. 4, pp. 1363-1369, 2005.

[23] Y. He, M. Wu, and J. H. She, "Delay-dependent exponential stability of delayed neural networks with time-varying delay," IEEE Transactions on Circuits and Systems II, vol. 53, no. 7, pp. 553-557, 2006.

[24] Y. He, G. P. Liu, D. Rees, and M. Wu, "Stability analysis for neural networks with time-varying interval delay," IEEE Transactions on Neural Networks, vol. 18, no. 6, pp. 1850-1854, 2007.

[25] T. Li, L. Guo, C. Sun, and C. Lin, "Further results on delaydependent stability criteria of neural networks with timevarying delays," IEEE Transactions on Neural Networks, vol. 19, no. 4, pp. 726-730, 2008.

[26] C. D. Zheng, H. G. Zhang, and Z. S. Wang, "Improved robust stability criteria for delayed cellular neural networks via the LMI approach," IEEE Transactions on Circuits and Systems II, vol. 57, no. 1, pp. 41-45, 2010.

[27] S. Mou, H. Gao, J. Lam, and W. Qiang, "A new criterion of delaydependent asymptotic stability for Hopfield neural networks with time delay," IEEE Transactions on Neural Networks, vol. 19, no. 3, pp. 532-535, 2008.

[28] H. Zhang, Z. Liu, G. B. Huang, and Z. Wang, "Novel weightingdelay-based stability criteria for recurrent neural networks with time-varying delay," IEEE Transactions on Neural Networks, vol. 21, no. 1, pp. 91-106, 2010.

[29] M. Wu, F. Liu, P. Shi, Y. He, and R. Yokoyama, "Exponential stability analysis for neural networks with time-varying delay," IEEE Transactions on Systems, Man, and Cybernetics B, vol. 38, no. 4, pp. 1152-1156, 2008.

[30] X. M. Zhang, M. Wu, J. H. She, and Y. He, "Delay-dependent stabilization of linear systems with time-varying state and input delays," Automatica, vol. 41, no. 8, pp. 1405-1412, 2005.

[31] H. Zhang, Z. Wang, and D. Liu, "Robust exponential stability of recurrent neural networks with multiple time-varying delays," IEEE Transactions on Circuits and Systems II, vol. 54, no. 8, pp. 730-734, 2007. 


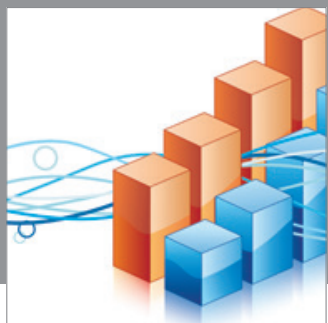

Advances in

Operations Research

mansans

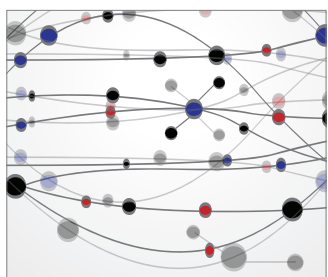

The Scientific World Journal
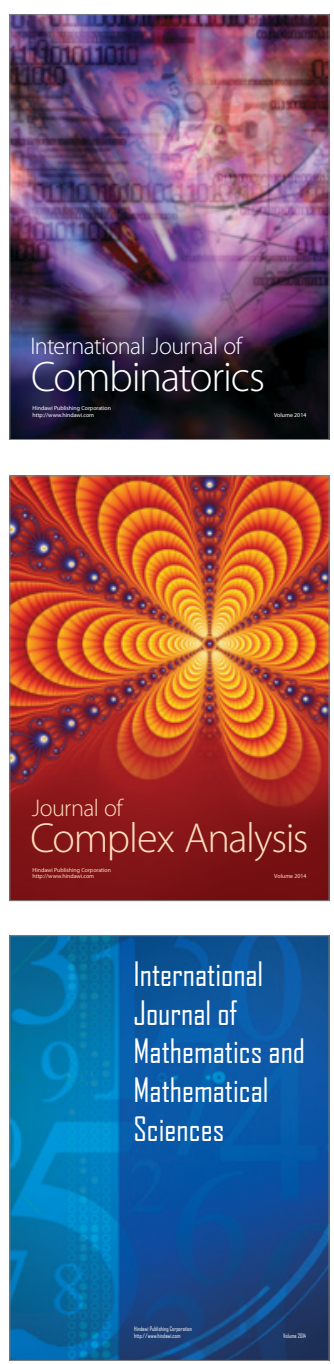
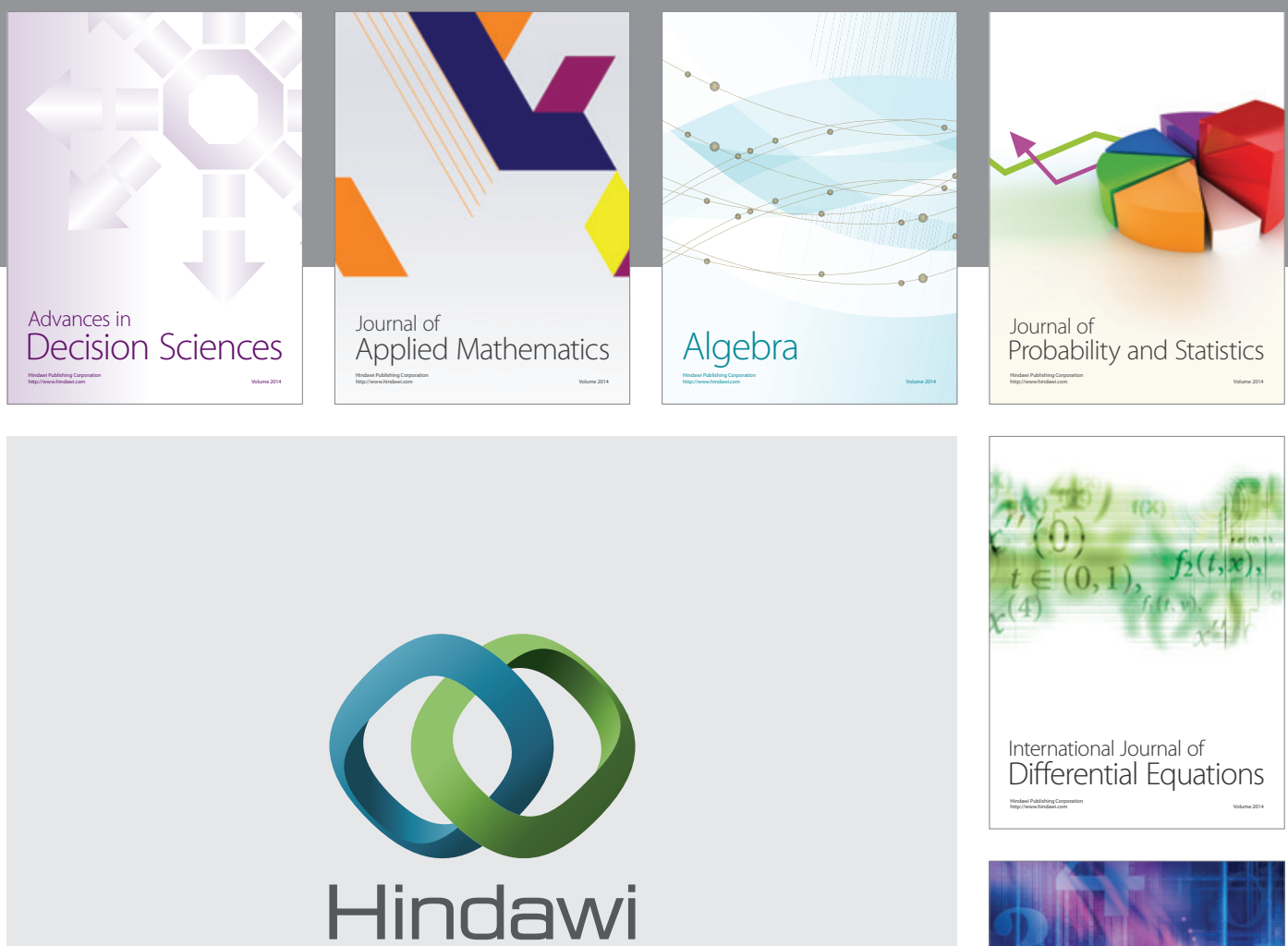

Submit your manuscripts at http://www.hindawi.com
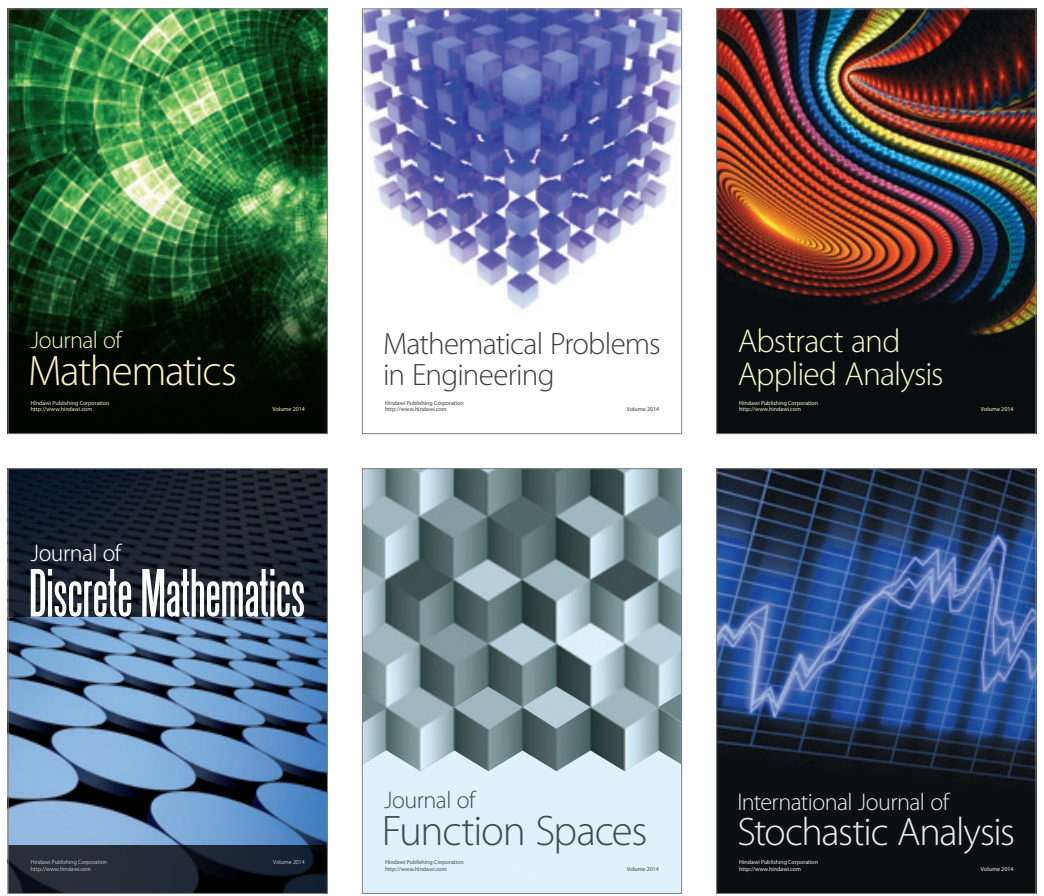

Journal of

Function Spaces

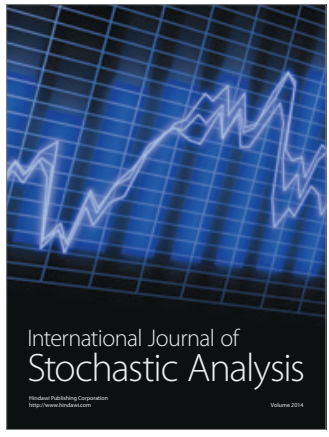

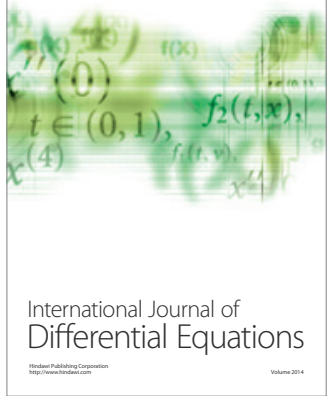
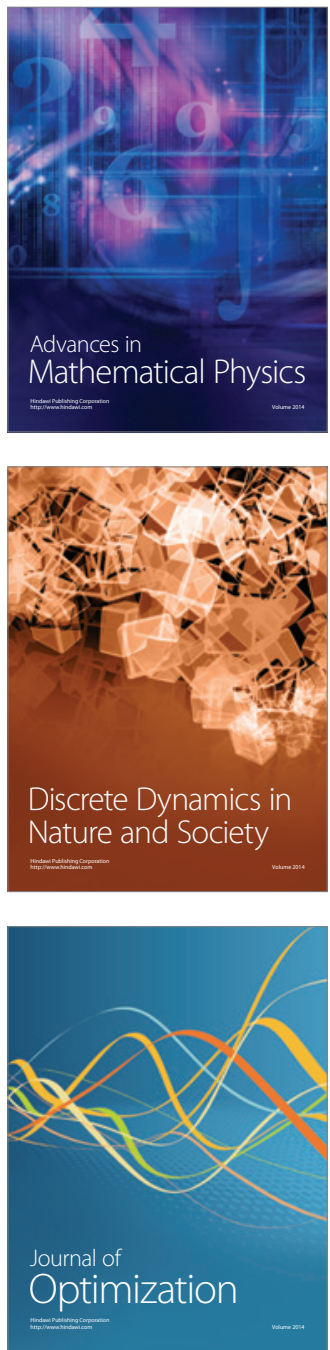